\title{
Two Bs or not two Bs? A signal detection theory analysis of repetition blindness in a counting task
}

\author{
CHRISTOPHER J. ANDERSON and W. TRAMMELL NEILL \\ State University of New York, Albany, New York
}

\begin{abstract}
Stimulus repetition usually benefits performance. A notable exception is repetition blindness (RB), in which subjects fail to report a repeated stimulus in a rapid serial visual presentation. Theories differ in attributing $\mathrm{RB}$ to either perceptual encoding or memory retrieval and to impaired discrimination versus response bias. In the present study, subjects judged whether one or two letters were imbedded in sequences of digits. Unlike previous studies, false guesses of two unrepeated letters were distinguished from false guesses of two repeated letters. When repeated- and unrepeated-letter trials were randomly intermixed (Experiment 1), RB was entirely attributable to response bias. However, when they were separately blocked (Experiments 2 and 3$)$, RB was manifested in discriminability $\left(d^{\prime}\right)$. The results support perceptual-encoding accounts of $\mathrm{RB}$ but indicate that effects on discriminability depend on subjects' processing strategies.
\end{abstract}

It seems nearly axiomatic that cognition should benefit from stimulus repetition. After all, repetitions of stimuli enhance memory performance (Ebbinghaus, 1885/1913), repeated stimuli are usually perceived more accurately (Haber, 1965; Haber \& Hershenson, 1965), and reaction time (RT) is usually faster to them (Bertelson, 1963; Keele, 1969). However, there are also a number of exceptions to this rule. For example, if a stimulus has recently been experienced as a distractor during the processing of another stimulus, RT to that stimulus is likely to be slower than RT to an unrepeated stimulus - that is, negative priming (Lowe, 1979; Neill, 1977; Neill \& Westberry, 1987; Tipper, 1985; Tipper \& Cranston, 1985). Similarly, preexposure of letter alternatives can impair tachistoscopic letter identification, the before-disruption effect (Neill, 1985; Neill \& Walling, 1981; Smith, Haviland, Reder, Brownell, \& Adams, 1976). Neill and Mathis (1998) have reviewed other examples in which the initial processing of a stimulus may be transfer inappropriate to the processing of a similar subsequent stimulus.

A particularly controversial performance decrement owing to repetition is the repetition blindness $(\mathrm{RB})$ phenomenon reported by Kanwisher (1987). In the most typical demonstration of $\mathrm{RB}$, a rapid sequence of visual stimuli is presented at a single spatial location. If two items in

Experiments 1 and 2 were part of a thesis submitted by the first author in partial fulfillment of the requirements for a master of arts degree at the University at Albany, State University of New York. We are indebted to Paul Haus, James Reilly, Suzanne Steinis, and Lauren Stutman for assistance with data collection and to James H. Neely for helpful comments during all phases of this work. Please direct correspondence regarding this report to W. T. Neill, Department of Psychology, University at Albany, SUNY, Albany, NY 12222 (e-mail: neill@csc.albany.edu). the sequence share the same identity, report of the second occurrence is often impaired, relative to an unrepeated item in the same serial position. This effect has been demonstrated with a variety of different materials, such as sequential words making up a sentence (Kanwisher, 1987), unrelated words (Bavelier \& Potter, 1992; Kanwisher, 1987), digits and letters (Armstrong \& Mewhort, 1995; Bavelier \& Potter, 1992; Chun \& Cavanagh, 1997; Luo \& Caramazza, 1995; Neill, Neely, Hutchison, Kahan, \& VerWys, 2002), pictures (Bavelier, 1994), complex nonsense shapes (Arnell \& Jolicœur, 1997), and simple shapes and colors (Kanwisher, 1991; Kanwisher, Driver, \& Machado, 1994, 1995).

Because RB has generally been demonstrated in a retrospective report of the stimulus sequence, much debate has centered on whether the effect is due to interference with perception or encoding of the repeated occurrence or, instead, to processes involved in subsequent retention or retrieval. Kanwisher (1987) initially proposed that RB occurs as a result of a failure to differentiate between different instances of the same type of event if they happen too closely togetherin time. More specifically, she argued that different instances of the same stimulus identity do successfully access their abstract conceptual representation in memory - that is, they both activate a common type node. However, the instances may fail to be encoded as separate tokens in episodic memory. Luo and Caramazza (1995) suggested that RB may, instead, be due to impaired categorization of the second instance-specifically, refractoriness of the type node.

Other researchers have argued that RB depends on either interference in memory or response bias at the time of report. Whittlesea, Dorken, and Podrouzek (1995; Whittlesea \& Podrouzek, 1995) argued that repeated instances 
are encoded no differently than unrepeated instances; however, subjects may fail to differentiate between instances of a repeated item at the time of retrieval. It is also possible that the overt report of the first occurrence of a repeated stimulus results in output interference with retrieval or report of the second, as in the Ranschburg effect in short-term memory (Crowder, 1968; Ranschburg, 1902).

Fagot and Pashler (1995) noted that a report of the first occurrence may also introduce a response bias against the second, for either of two reasons: (1) If subjects fail to recognize the second stimulus, they may tend to guess an item not yet reported (guessing bias); (2) when repeated stimuli are both recognized, subjects may still be reluctant to emit the same response twice (censorship bias). Consistent with either output interference or response bias, Fagot and Pashler (1995, Experiment 1) reported that RB was eliminated if subjects were required to report the list items in reverse order.

In general, proponents of memory retrieval accounts of RB have emphasized the effects of manipulations at the time of report on the magnitude of RB. Several studies (Armstrong \& Mewhort, 1995; Fagot \& Pashler, 1995; Lewandowsky, Neely, VerWys, \& Amos, 1997) have found $\mathrm{RB}$ to be eliminated if recall of the second occurrence of a repeated item was specifically cued. For example, Fagot and Pashler (1995, Experiment 4) presented sequences of six letters, in which one was colored red. After a sequence, the subject was cued to either first report the red letter or first report the whole sequence. Whole report yielded RB for the second occurrence of a repeated letter, even if it was the one shown in red. However, if the subjects were cued to first report the red letter, report of a repeated letter did not differ from report of an unrepeated letter. On the other hand, some studies have found RB with a cued report, even with minimal memory load (Hochhaus \& Johnston, 1996; Hochhaus \& Marohn, 1991; Luo \& Caramazza, 1995; Neill et al., 2002).

Park and Kanwisher (1994, Experiment 7) attempted to rule out memory retrieval as an explanation for RB by using a task in which subjects had to count whether one or two vowels appeared in a sequence of letters. The essential logic was that subjects had to individuate occurrences but no report of stimulus identities was required. The memory demands for a count of "two" should be identical for two identical vowels or two nonidentical vowels, and of course, no output interference could occur. Although it would still be possible that the subjects could adopt a bias against reporting "two" when two identical letters were shown, Park and Kanwisher used a signal detection theory (SDT) analysis (Green \& Swets, 1966; Macmillan \& Creelman, 1991) to measure sensitivity independently of response bias. Sensitivity, as measured by $d^{\prime}$, was significantly reduced for two identical letters, relative to two nonidentical letters.

Kanwisher, Kim, and Wickens (1996) recognized a potential problem in the Park and Kanwisher (1994) study: that categorization of a letter as a vowel might be sufficiently difficult that postidentification processes might cause interference between repetitions. They therefore ran a modified procedure in which two possible target identities were specified at the beginning of a block. For example, the subject might be informed that $\mathrm{A}$ and $\mathrm{E}$ were the possible vowels; a repeated trial would therefore contain two As or two Es, an unrepeated trial would contain an A and an $\mathrm{E}$ (in either order), and a single-vowel trial would contain one A or one E. In addition, they conducted a replication of the more abstract vowel detection task used by Park and Kanwisher. Both tasks yielded similar results: $d^{\prime}$ (as well a high-threshold alpha measure) was lower for two repeated vowels than for unrepeated identities. ${ }^{1}$

A problem with these analyses, however, is that Kanwisher et al. (1996; Park \& Kanwisher, 1994) could not determine whether false "two" responses resulted from guesses (or misperceptions) that two identical vowels had occurred or from guesses (or misperceptions) that two different vowels had occurred. That is, the same false alarm rate on single-letter trials was used for the calculation of $d^{\prime}$ for both repeated-vowel and unrepeated-vowel conditions. Thus, a difference in $d^{\prime}$ could be determined only by a difference in hit rates. The difficulty with such an analysis is demonstrated by the following thought experiment.

Suppose that repeated and unrepeated conditions yielded identical hit rates (correct "two" responses) but that substantial false alarms (incorrect "two" responses) also occurred on single-vowel trials. An SDT analysis using the common false alarm rate would yield identical $d^{\prime}$ measures for repeated and unrepeated vowels. But if the subjects' false alarms occurred only because they thought that they saw the same vowel twice, their discrimination between two identical targets versus one target must be worse than their discrimination between two nonidentical targets versus one-that is, RB. However, if false alarms were never due to guesses that the same vowel was repeated, the subjects' ability to discriminate between two repeated targets versus one must be better than their discrimination between two unrepeated targets versus onethat is, repetition priming. Even in the presence of a significant difference between hit rates, the nature of the false alarms might well reverse the conclusions about which condition affords better discriminability.

In the present experiments, subjects were required to report the number of letters that were imbedded in a sequence of digits, one or two. We attempted to differentiate false alarms in two different ways. In Experiment 1, the subjects were given three response options: "one letter," "two identical letters," or "two different letters." In Experiments 2 and 3, we separately blocked the repeatedletter and unrepeated-letter conditions. The subjects were explicitly informed, at the beginning of some blocks, that two-letter trials would have two identical letters and, at the beginning of other blocks, that two-letter trials would have two different letters (and response options after each trial were similarly constrained). Here, a false "two" report on a single-letter trial would unambiguously imply a guess (or misperception) corresponding to the only possible two-letter relation, identical or nonidentical. 
Table 1

Response Proportions to Single-Letter, Repeated-Letter, and Unrepeated-Letter Trials in Experiment 1

\begin{tabular}{lcccr}
\hline & \multicolumn{3}{c}{ Response } \\
\cline { 2 - 4 } \multicolumn{1}{c}{ Trial Type } & One & Repeated & Unrepeated & Total \\
\hline Single letter & $.70(\mathrm{a})$ & $.08(\mathrm{~b})$ & $.22(\mathrm{c})$ & 1.00 \\
Repeated letter & $.29(\mathrm{~d})$ & $.42(\mathrm{e})$ & $.29(\mathrm{f})$ & 1.00 \\
Unrepeated letter & $.22(\mathrm{~g})$ & $.07(\mathrm{~h})$ & $.71(\mathrm{i})$ & 1.00 \\
\hline
\end{tabular}

Note-The means are collapsed over presentation rates. The cells are labeled here to facilitate discussion of different possible signal detection models in the text.

\section{EXPERIMENT 1}

\section{Method}

Subjects. We tested 61 University at Albany undergraduates, who participated to partially fulfill a requirement in an introductory psychology course. One subject was discarded from the final sample for failure to meet an a priori exclusion criterion of $60 \%$ overall accuracy. All subjects reported normal or corrected-to-normal vision. Each was tested individually in a session lasting 20-25 min.

Apparatus and Stimuli. Stimuli were presented on a Vivitron 1572 color monitor controlled by a Gateway 2000 PC-compatible microcomputer. Responses were made on the S, N, and K keys of the computer keyboard, which were labeled with red, blue, and green stickers, respectively. The experiment was programmed in Version 2.0 of Micro Experimental Laboratory (MEL; Schneider, 1988). The target stimuli were the capital letters A, B, C, and D, and the nontarget stimuli were digits 1-9, all in the default MEL letter font.

Procedure. At the beginning of each trial, the prompt "READY?" was displayed until the subject pressed the space bar to initiate the trial. After a 500-msec blank period, a fixation cross appeared in the center of the screen for $500 \mathrm{msec}$. This was then replaced by a rapid sequence of 10 digits and letters in the same location, at $83 \mathrm{msec} /$ item for 30 subjects and $100 \mathrm{msec} /$ item for 30 subjects. On singleletter trials, a letter randomly selected from A, B, C, or D appeared at either Position 4 or 7 , with a digit appearing at the other position. On repeated-letter trials, the same letter appeared at both positions, and on unrepeated-letter trials, different letters appeared at these two positions. Randomly selected digits occupied all the other positions, with the constraint that no digit was repeated in the sequence. Singleletter, repeated-letter, and unrepeated-letter sequences each appeared with one-third probability.

After each stimulus sequence, the subjects were presented with a response menu indicating that they should press the red key if they thought they had seen only one letter, the blue key if two identical letters, or the green key if two different letters. In order to minimize the effects of sequential response preferences, the order of choices was reversed for half of the subjects. A computer-generated tone signaled an incorrect choice.

After receiving instructions at the beginning of the experiment, the subjects received 10 practice trials at $133 \mathrm{msec} /$ item, to familiarize them with the procedures. They then participated in 144 experimental trials.

\section{Results}

In the analyses reported below, we included presentation rate as a between-subjects variable and repetition condition (repeated or unrepeated targets) as a within-subjects variable in $2 \times 2$ analyses of variance (ANOVAs). Unsurprisingly, performance was higher in the longer exposure duration. However, with one minor exception noted in Analysis 3 below, exposure duration did not interact sig- nificantly with repetition condition in the SDT analyses. Because we had no a priori predictions regarding this variable, we will simplify the presentation here by omitting discussion of duration effects. ${ }^{2}$ Table 1 shows the response proportions for each condition, collapsed over exposure duration, labeled (a) through (i) to facilitate the discussion of different signal-detection models below.

It may first be noted that the overall proportion correct is much higher in the unrepeated condition (.71) than in the repeated condition $\left[.42 ; F(1,58)=183.13, M S_{\mathrm{e}}=0.013\right.$, $p<.001]$. Thus, superficially, it appears that a very strong RB effect was obtained. However, in the single-letter condition, it is also evident that errors were much more likely to be due to a misreport of two different letters (.22) than of two identical letters $\left[.08 ; F(1,58)=134.65, M S_{\mathrm{e}}=\right.$ $0.004, p<.001]$. This result alone justifies the concern that the source of false "two" responses cannot be ignored in the determination of whether RB is really due to impaired discriminability of repeated letters. That is, subjects rarely guess that two identical letters have been shown, and so the ability to discriminate two versus one letter might, in fact, be very good for repeated letters, despite a low hit rate.

We conducted three different SDT analyses, to illustrate the consequences of assumptions made regarding false "two" responses. In the analyses that follow, it will be useful to keep in mind that the SDT measure of discriminability, $d^{\prime}$, is only a measure of dichotomous discriminability - that is, discriminability between " $X$ " and "not $X$ " (or between "X present" and "X not present"). Because there are three possible responses, rather than just "present" or "absent," the nature of "not X" becomes critical to interpreting any effect on $d^{\prime}{ }^{3}$

Analysis 1. In our first analysis, we demonstrated the consequences of not differentiating between sources of false alarms; that is, we treated all "two" responses as the same. This is equivalent to the analysis conducted by Park and Kanwisher (1994) and Kanwisher et al. (1996). With regard to Table 1, this entailed adding Cell (b) to (c), (e) to (f), and (h) to (i). The mean hit rates (correct "two" judgments) were then .71 for the repeated condition and .78 for the unrepeated condition. The mean false alarm rate (false "two" judgment) was .30. After applying this transformation to individual subject data, mean $d^{\prime}$ was significantly lower in the repeated condition (.28) than in the unrepeated condition $\left[1.15 ; F(1,58)=137.30, M S_{\mathrm{e}}=\right.$ $0.167, p<.001]$. Thus, if we treat all "two" responses as equivalent, there appears to be a strong RB effect.

No effects on the SDT measure of response bias, $\beta$, were significant (all $F \mathrm{~s}<1.5){ }^{4}$

Analysis 2. Here, we asked how the discrimination of repeated targets versus the other conditions (unrepeated and single target) differed from discrimination of unrepeated targets versus the other conditions (repeated and single target). With regard to Table 1, this entailed averaging Cells (b) and (h) for a mean false alarm rate of .08, combined with a hit rate of .42 for repeated-target trials, and averaging Cells (c) and (f) for a mean false alarm rate of 
.26 , to be combined with a hit rate of .71 for unrepeatedtarget trials. Here, the difference in $d^{\prime}$ calculated for repeated targets (1.24) and for unrepeated targets (1.34) failed to achieve significance $[F(1,58)=2.38, p>.10]$. On the other hand, the response criterion was significantly higher for repeated-target responses (mean $\beta=2.54$ ) than for unrepeated-target responses $[1.07 ; F(1,58)=47.46$, $\left.M S_{\mathrm{e}}=2.99, p<.001\right]$, indicating a relative bias against making the "two identical" response.

Analysis 3. Here, we attempted to ask how repeated and unrepeated conditions differed in discriminability from the single-target condition, taking into account the different false alarm rates for the 2 two-target conditions. We reasoned as follows. For calculating the discrimination of repeated targets from single targets, both the unrepeatedtarget condition and any "two different" responses would be irrelevant-we would pretend that they do not exist. Hence, in reference to Table 1, only Cells (a), (b), (d), and (e) would be relevant. Similarly, for calculating the discrimination of unrepeated targets from single targets, only cells (a), (c), (g), and (i) would be relevant. However, an SDT analysis assumes that hits and misses sum to one and that correct rejections and false alarms sum to one. It was therefore necessary to conditionalize the proportions as follows: hit rate $($ repeated condition $)=(\mathrm{e}) /[(\mathrm{d})+(\mathrm{e})]=$ .59; false alarm rate (repeated condition) $=(\mathrm{b}) /[(\mathrm{a})+$ (b) $]=.10$; hit rate $($ unrepeated condition $)=(\mathrm{i}) /[(\mathrm{g})+$ (i) $]=.76$; false alarm rate (unrepeated condition) $=(\mathrm{c}) /$ $[(\mathrm{a})+(\mathrm{c})]=.24$. (The proportions here are approximate, based on the Table 1 cell means for illustrative purposes. The actual means differed slightly, owing to variability of the individual base rates used to conditionalize the proportions.)

After applying these transformations to the individual subject data, $d^{\prime}$ for the repeated condition (1.57) did not differ significantly from $d^{\prime}$ for the unrepeated condition $[1.52 ; F(1,58)<1]$. Again, there was no evidence for an $\mathrm{RB}$ effect on discriminability. Here, repetition condition did interact significantly with exposure duration $[F(1,58)=$ $\left.4.18, M S_{\mathrm{e}}=0.202\right]$. In the 83-msec group, $d^{\prime}$ was slightly higher for unrepeated targets (1.22) than for repeated targets (1.10), but in the 100-msec group, this pattern was reversed (1.82 vs. 2.04). Neither simple effect was statistically significant. On the other hand, the response criterion was again higher for repeated-target responses (mean $\beta=2.14$ ) than for unrepeated-target responses [.99; $\left.F(1,58)=16.24, M S_{\mathrm{e}}=0.021, p<.001\right]$.

\section{Discussion}

These results clearly illustrate the importance of distinguishing between "two" responses that are due to guesses of two identical targets and "two" responses that are due to guesses of two unidentical targets. When all "two" responses were treated as equivalent (Analysis 1), the results provided a direct replication of Park and Kanwisher (1994) and Kanwisher et al. (1996): Not only was the proportion of "two" judgments lower for repeated-target trials than for unrepeated-target trials, the SDT measure $d^{\prime}$ was also significantly lower for repeated-target trials. However, given the first result, the second is unsurprising, because exactly the same false alarm rates were used in the calculation of $d^{\prime}$. But it is obvious from the obtained response proportions in the single-target condition that the subjects were much less likely to guess that they had seen two repeated targets than to guess that they had seen two different targets, and so this result must be viewed as highly suspect.

When we used the different false alarm rates for "two identical" and "two different" responses (Analyses 2 and 3), the difference in $d^{\prime}$ between repeated and unrepeated conditions evaporated, and highly significant differences emerged, instead, in response bias, $\beta$. In other words, the difference in correct detections of two unrepeated letters versus two repeated letters could be attributed entirely to the reluctance of the subjects to guess that there were two repeated letters.

In a standard SDT analysis, dichotomous responses (" $\mathrm{X}$ present" or "X not present") are conditionalized on dichotomous stimulus conditions (X present or X not present). In this experiment, there were three possible responses and three stimulus conditions. Therefore, it is important to consider how nine cells should be condensed into a $2 \times 2$ matrix for analysis. Some comment is therefore warranted on the assumptions made in Analyses 2 and 3.

We believe that Analysis 2 is preferable for testing the hypotheses of concern. Here, we compared $d^{\prime}$ for repeated targets versus "other" with $d^{\prime}$ for unrepeated targets versus "other." The critical observation here is that the discriminability of repeated from unrepeated targets contributes equally to both $d^{\prime}$ values. Therefore, a difference in $d$ 'should reflect only the difference in discriminability of two identical targets from one target, as compared with the discriminability of two nonidentical targets from one target. The only limitation is that the absolute values of $d^{\prime}$ do not estimate the discriminability of each two-target condition from the single-target condition alone. ${ }^{5}$

In Analysis 3, we attempted to do this more directly but also made more assumptions in doing so. In particular, we supposed that, for the calculation of $d^{\prime}$ for repeated letters, we could ignore the unrepeated-letter condition and any "two different" responses and that, for the calculation of $d^{\prime}$ for unrepeated letters, we could ignore the repeated-letter condition and any "two identical" responses. As such, each $d^{\prime}$ and $\beta$ might ignore potentially informative data. We took comfort, however, in the fact that this analysis afforded essentially the same conclusion as Analysis 2 - that is, a significant difference in response bias, but not in discriminability (both opposite to Analysis 1).

Both Analyses 2 and 3 might be assailed on the grounds that they deviated from the usual assumptions of SDT, insofar as a fundamentally $2 \times 2$ analysis was used to interpret data from a $3 \times 3$ design. However, the most important result of this experiment cannot be overstated: On single-letter trials, the subjects were much more likely to guess "two different" than "two identical." Therefore, any analysis that ignores the source of false "two" reports 
(e.g., Kanwisher et al., 1996; Park \& Kanwisher, 1994) is rendered suspect.

Finally, we should consider whether the design of this experiment might have directly mitigated against a "genuine" RB effect (i.e., a $d^{\prime}$ effect). Repeated-letter and unrepeated-letter trials were equal in frequency. Therefore, given the identification of one letter, the probability that a second letter would be the same letter was three times higher than that for any one of the other three possible letters. Thus, it is possible that stimulus uncertainty effects might have favored the repeated condition, apart from any response bias effects. This concern was directly addressed in Experiments 2 and 3.

\section{EXPERIMENTS 2 AND 3}

In Experiments 2 and 3, repeated-letter and unrepeatedletter trials were presented in separate blocks. Accordingly, a false "two" response on a single-letter trial could be unambiguously attributed to a guess of the only possible type of two-letter trial. The SDT analyses were therefore more straightforward than those in Experiment 1, because independent false alarm rates were available for the repeated and the unrepeated conditions. As in Experiment 1, stimulus uncertainty, conditional on identification of one letter, was again necessarily greater in the unrepeatedletter condition than in the repeated-letter condition, which could mitigate against finding an RB effect. However, to anticipate the results, we found strong RB effects, in contrast to Experiment 1. Experiment 3 was run essentially as a replication of Experiment 2, in order to ensure that the markedly different results from Experiment 1 were not due to differences in apparatus or display characteristics.

\section{Method}

Subjects. University at Albany undergraduates participated in partial fulfillment of requirements in an introductory psychology course (38 in Experiment 2 and 26 in Experiment 3). In Experiment 2,8 subjects were eliminated from the final sample for failure to meet an a priori exclusion criterion of $60 \%$ overall performance. All the subjects reported normal or corrected-to-normal vision. Each was tested individually in a session lasting 20-25 min.

Stimuli and Apparatus. In Experiment 2, the stimuli were presented on a monochrome monitor controlled by an Apple IIe microcomputer. In Experiment 3, they were presented on a Vivitron 1572

Table 2

Proportions of Correct "One" or "Two" Judgments on One-Letter and Two-Letter Trials as a Function of Repetition Condition (Experiments 2 and 3)

\begin{tabular}{ccc}
\hline Condition & Experiment 2 & Experiment 3 \\
\hline Repeated & & .79 \\
One letter & .66 & .63 \\
Two letter & .56 & \\
Unrepeated & & .75 \\
One letter & .62 & .77 \\
Two letter & .73 &
\end{tabular}

Note-The proportions for Experiment 2 are collapsed over presentation rate. Presentation rate was not varied in Experiment 3. color monitor controlled by a Gateway 2000 microcomputer, as in Experiment 1. Experiment 2 was programmed in Applesoft Basic, and Experiment 3 was programmed in MEL. The same stimulus sets were used as those in Experiment 1.

Procedure. The major procedural difference from Experiment 1 was that repeated-letter and unrepeated-letter trials appeared in separate blocks. At the beginning of each block, the subjects were informed either that two-letter trials would repeat the same letter or that they would display different letters. In addition, at the end of each stimulus sequence, the subjects were prompted to respond whether they had seen "one letter or two of the same letter" (for repeatedletter blocks) or "one letter or two different letters" (for unrepeatedletter blocks). Only two keyboard keys, labeled " 1 " and " 2 ," were used to record responses.

The subjects received two practice blocks, one in each condition. The practice sequences were presented at a slow rate $(333 \mathrm{msec} / \mathrm{item}$ in Experiment 2, $133 \mathrm{msec} /$ item in Experiment 3) to ensure that the subjects understood the two sets of instructions. For the first 23 subjects in Experiment 2, the stimuli were presented at $83 \mathrm{msec} /$ item in the experimental blocks. Because our exclusion rule of $60 \%$ overall accuracy forced us to reject 8 subjects, we tested 15 more subjects at $100 \mathrm{msec} /$ item. In Experiment 3, all the subjects received an exposure duration of $100 \mathrm{msec} /$ item. The subjects participated in $10 \mathrm{ex}-$ perimental blocks of 15 trials each. Repeated-target and unrepeatedtarget blocks were alternated, with order counterbalanced over subjects.

\section{Results}

Table 2 displays the mean proportion correct for oneand two-target trials in Experiments 2 and 3 as a function of repetition condition. For each experiment, we first will report an analysis of these proportions, followed by an SDT analysis of $d^{\prime}$ and $\beta$ effects.

Experiment 2. The proportions of correct responses were submitted to a $2 \times 2 \times 2$ ANOVA with a betweensubjects variable of exposure duration and within-subjects variables of repetition condition and trial type (one or two letters). Overall performance was significantly worse in the repeated condition (.61) than in the unrepeated condition $\left[.68 ; F(1,28)=20.17, M S_{\mathrm{e}}=0.007, p<.001\right]$, and repetition interacted significantly with trial type $[F(1,28)=$ $\left.42.01, M S_{\mathrm{e}}=0.008, p<.001\right]$. Planned comparisons indicated that accuracy was significantly worse for two repeated targets (.56) than for two unrepeated targets [.73; $t(28)=8.34, p<.001$, two-tailed]. In contrast, accuracy was marginally better for one-target trials in the repeated condition (.66) than in the unrepeated condition [.62; $t(28)=1.99, p<.10$, two-tailed]. Thus, the proportions of correct "two" responses again suggest an RB effect, but the opposite-direction difference for "one" responses again raises the possibility that this result could be due to a greater reluctance to respond "two" in the repeated condition.

An SDT analysis indicated a significantly higher $d^{\prime}$ in the unrepeated condition (.99) than in the repeated condition $\left[.59 ; F(1,28)=21.39, M S_{\mathrm{e}}=0.336, p<.001\right]$. In addition, the response criterion was higher for repeated trials $(\beta=1.09)$ than for unrepeated trials $[0.85 ; F(1,28)=$ $\left.16.08, M S_{\mathrm{e}}=0.024, p<.001\right]$.

Experiment 3. The proportions of correct responses were entered into a $2 \times 2$ ANOVA with within-subjects 
variables of repetition condition and trial type. All effects were statistically significant: Performance was higher overall in unrepeated-letter blocks (.75) than in repeatedletter blocks $\left[.71 ; F(1,25)=7.71, M S_{\mathrm{e}}=0.010, p<.01\right]$ and on one-letter trials (.77) than on two-target trials [.70; $\left.F(1,25)=5.32, M S_{\mathrm{e}}=0.023, p<.05\right]$. Repetition condition interacted with trial type $\left[F(1,25)=29.22, M S_{\mathrm{e}}=\right.$ $0.007, p<.001]$, reflecting an advantage of twounrepeated trials (.77) over two-repeated trials (.63), but a reversal for the corresponding one-target trials (.75 vs. .79). Planned comparisons indicated that the first simple effect was significant $[t(25)=5.055, p<.001]$, whereas the second was not.

SDT analyses indicated that $d^{\prime}$ was significantly higher for the unrepeated condition (1.58) than for the repeated condition $[1.22 ; t(25)=2.74, p<.02$, two-tailed]. In addition, the response criterion was higher for the repeated condition $(\beta=1.28)$ than for the unrepeated condition $[0.92 ; t(25)=2.65, p<.02$, two-tailed $]$.

\section{Discussion}

As in Experiment 1, the proportions of correct "two" responses (i.e., hit rates) were higher for two unrepeated letters than for two repeated letters, but the false "two" guesses also tended to be higher for the unrepeated-letter condition. However, the results of Experiments 2 and 3 were markedly different from those of Experiment 1, in that the SDT measure of discriminability, $d^{\prime}$, was significantly higher in the unrepeated condition than in the repeated condition. That is, the difference in false alarm rates was not enough to account for the difference in hit rates. Thus, both experiments support a genuine RB effect that cannot be attributed to response bias.

Why did Experiments 2 and 3 yield a genuine RB effect, whereas Experiment 1 did not (when differential false alarm rates were taken into account)? It was noted in the discussion of Experiment 1 that the equal probability of repeated and unrepeated trials necessarily resulted in greater stimulus uncertainty for an unrepeated letter (three possible letters) than for a repeated letter (only one), conditionalizedon identification of the other letter. This would favor the repeated condition, relative to the unrepeated condition. Thus, it is possible that Experiment 1 would have yielded an RB effect if stimulus uncertainty had been equated (e.g., if the total possible stimulus set consisted of only two letters). However, this same statistical bias against an RB effect was present in Experiments 2 and 3 and so cannot account for the difference in results.

It seems more likely that the different results in Experiment 1 versus Experiments 2 and 3 were caused either by the difference in response requirements (three choices vs. two) or by effects of randomization versus blocking of trial types. It is possible that the requirement to choose between two identical letters versus two different letters (in Experiment 1) induced the subjects to attend more to the contextual information (e.g., list position) that would help to distinguish between the first and the second occurrences of a repeated letter. Alternatively, it is possible that the subjects were more vigilant for repetitions than for nonrepetitions in Experiment 1, even though repeated and unrepeated two-letter trials were equal in objective likelihood. This would not have occurred in Experiments 2 and 3, because there was no uncertainty about the nature of twoletter trials.

\section{GENERAL DISCUSSION}

In the present experiments, the subjects were required to judge whether one or two letters were imbedded in a rapid sequence of digits. Previous research in which a similar counting task was used (Kanwisher et al., 1996; Park \& Kanwisher, 1994) found a significant decrement in discriminability (as measured by $d^{\prime}$ ) if two letters in a sequence were identical - that is, an apparent RB effect. However, the previous studies were flawed in their failure to distinguish between guesses (or misperceptions) of two identical targets versus two different targets. The present Experiment 1 demonstrated that subjects are much less likely to guess that two identical targets had been shown than to guess that two different targets have been shown. Although correct detections of two identical targets were much lower than correct detections of two different targets, SDT analyses that took into account the guessing biases confirmed that differential response bias could, in fact, account for the apparent RB effect.

On the other hand, Experiments 2 and 3 indicated that guessing bias cannot fully account for RB effects. Here, trials with two repeated letters or two unrepeated letters were presented in separate blocks (with one-letter trials). As in Experiment 1, correct detections of two repeated letters were fewer than correct detections of two unrepeated letters, and there were also fewer guesses of two repeated letters than of two unrepeated letters. Unlike Experiment 1 , however, the difference in guessing rates was not enough to account for the difference in detection rates: Both experiments found significantly lower $d^{\prime}$ for detecting two repeated letters.

As was discussed in the introduction, there has been considerable debate over whether RB is due to impaired perceptual encoding of repetitions or to interference at time of report and over whether the effect is due to reduced information (a $d^{\prime}$ effect) or only to response bias. We hasten to point out that these two issues are orthogonal: Just as either $d^{\prime}$ or $\beta$ effects can occur during perceptual classification, so either can occur owing to factors that influence memory retrieval. For example, test delay and targetdistractor similarity both reduce $d^{\prime}$ in recognition memory tests (Murdock, 1974), but neither effect could possibly be attributed to perceptual encoding processes. Rather, it is here the presence of a $d^{\prime}$ effect in experiments designed to exclude memory factors that compels the conclusion that RB is due to impaired encoding of repeated instances.

Kanwisher (1987, 1991; Kanwisher et al., 1996; Park \& Kanwisher, 1994) has argued that repeated stimuli are both perceptually categorized, in the sense that both access their abstract representation in memory; that is, they 
activate their common type node. However, they may fail to be perceived as separate instances, and so only one token is encoded for both events. Luo and Caramazza (1995) also argued for a perceptual encoding effect but attributed $\mathrm{RB}$ to refractoriness of the type node; that is, the second occurrence may not be perceptually categorized. Either theory can accommodate the present results. However, both tokenization failure and type refractoriness have difficulties accounting for other findings-notably, that RB can occur for the first occurrence of a repeated target as strongly (or more strongly) than for the first occurrence (Neill et al., 2002).

Most researchers have assumed that RB is a proactive effect-that is, the first occurrence somehow interferes with the processing of the second, but not vice versa. This assumption is inherent in the perceptual accounts of RB put forth by Kanwisher (1987) and Luo and Caramazza (1995). Kanwisher (1987) has argued specifically that it is the creation of a token for the first stimulus that interferes with creation of a second token of the same type. Similarly, type refractoriness (Luo \& Caramazza, 1995) could occur only if the first occurrence has successfully activated its type. However, it is surprisingly difficult to ascertain from the literature whether an RB effect does occur for the first of two repeated instances: Some studies have reported the performance only on the second occurrence, or they have reported the conjunctive probability that both stimuli were identified. In many studies, output order was confounded implicitly or explicitly with presentation order, which may have made RB on the second occurrence more apparent. Or, whereas stimulus materials might have been counterbalanced for the second occurrence, they may not have been for the first (see Neill et al., in press, for more detailed discussion) .

Neill et al. (2002) conducted a series of experiments in which two letters were presented sequentially to the left and right of fixation, followed by pattern masks. By chance (25\%), the two letters could be identical. In some conditions, subjects were cued to report the letter that appeared at a specified location; in some, they were cued to report the letter that appeared in a specified temporal position (first or second). The results were inconsistent with nearly all proposed theories of RB: All six experiments showed substantial RB on the first occurrence; furthermore, RB was greater for the first occurrence than for the second if the subjects expected spatial cuing but was greater on the second if they expected temporal cuing. There were no interactions of RB with output position, and analyses of guessing responses on error trials and catch trials (with only one presented letter) indicated a general bias toward, rather than against, guessing repeated letters.

Neill et al. (2002) suggested that Kanwisher's (1987) tokenization theory could be modified to account for the results by distinguishing between the formation of a token (instantiation) and the assignment of that token to a particular spatial or temporal context (attribution). Thus, in agreement with Kanwisher (1987), the failure to distinguish between two instances of the same letter would re- sult in only one episodic representation, or token, for that letter. However, it would still be necessary to associate the token with a spatial location and/or temporal position. Neill et al. (2002) suggested that ecological constraints favor associating an object with its most recent occurrence if its location is of primary importance, but with its first occurrence if its temporal position is of primary importance. Thus, RB should be greater for the first occurrence if subjects are attending to spatial location, but greater on the second occurrence if they are attending to temporal position.

The present data cannot discriminate between RB for the first presented letter versus RB for the second-an incorrect "one" response could result from a failure to identify either the first or the second occurrence. Nonetheless, the data are consistent with the modification of Kanwisher's (1987) tokenization failure theory proposed by Neill et al. (2002; Neill \& Mathis, 1998). That is, if only one token is encoded for repeated letters, it makes little difference whether that token is attributed to the earlier or the later list position; the encoding of only one token should result in an incorrect "one" response.

We acknowledge that the conclusion of a perceptual/ encoding RB effect here does not preclude the possibility that RB effects can also be caused by either guessing biases or memory retrieval factors. Indeed, we concluded that an apparent RB effect in Experiment 1 was due to guessing biases, rather than to discriminability. Furthermore, a majority of RB studies have used near-span whole report of multiple items. In such procedures, there is ample opportunity for retention and retrieval factors to interact with repetitions in report. However, given that repetition can cause perceptual decrements in very simple displays with minimal memory demands, those same decrements are also likely to affect performance in more complex tasks. It will then remain a challenge for investigators to adequately distinguish between on-line repetition effects and off-line repetition effects that may be superimposed on them.

As was noted at the beginning of this paper, it often seems near-axiomatic that performance should benefit from stimulus repetition. However, as has been reviewed by Neill and Mathis (1998), there are numerous exceptions in perceptual identification, reaction time, and memory paradigms. A critical variable may be whether the processing required for the first instance is consistent with the processing required for the second instance-that is, whether the processing is transfer appropriate or transfer inappropriate. Superficially, in the procedures used to demonstrate $\mathrm{RB}$, processing requirements for the first and the second occurrences of a stimulus seem to be the samenamely, the occurrences must be both attended and identified. However, the processing requirements are really not the same: One instance must be assigned to one spatial or temporal context, whereas the second must be assigned to a different context.

Although transfer appropriateness has played a major role in theoretical accounts of facilitation by repetition (Blaxton, 1989; Morris, Bransford, \& Franks, 1977; Roedi- 
ger \& Blaxton, 1987), relatively little attention has been devoted to the consequences of transfer inappropriateness. It is often difficult to anticipate in advance whether the processing requirements for repeated stimuli are, on the whole, more similar or more dissimilar. Indeed, the judgment of overall transfer appropriateness or inappropriateness is entirely post hoc, depending on whether facilitation or impairment occurs as a result of repetition. Nonetheless, just as an orientation toward transfer appropriateness has led researchers to useful questions of how processing requirements are similar across repetitions, an orientation toward transfer inappropriateness leads to questions of how processing requirements are dissimilar across repetitions. In principle, this should facilitate the description of what perceptual and cognitive processes in fact underlie performance in a given task.

\section{REFERENCES}

Armstrong, I. T., \& Mewhort, D. J. K. (1995). Repetition deficit in rapid-serial-visual-presentation displays: Encoding failure or retrieval failure? Journal of Experimental Psychology: Human Perception \& Performance, 21, 1044-1052.

Arnell, K. M., \& Joliceur, P. (1997). Repetition blindness for pseudo-object pictures. Journal of Experimental Psychology: Human Perception \& Performance, 23, 999-1013.

Bavelier, D. (1994). Repetition blindness between visually different items: The case of pictures and words. Cognition, 51, 199-236.

Bavelier, D., \& Potter, M. C. (1992). Visual and phonological codes in repetition priming. Journal of Experimental Psychology: Human Perception \& Performance, 18, 134-137.

Bertelson, P. (1963). S-R relationships and reaction times to new versus repeated signals in a serial task. Journal of Experimental Psychology, $\mathbf{6 5}, 478-484$.

BlaXton, T. A. (1989). Investigating dissociations among memory measures: Support for a transfer-appropriate processing framework. Journal of Experimental Psychology: Learning, Memory, \& Cognition, 15, 657-668.

Chun, M. M., \& Cavanagh, P. (1997). Seeing two as one: Linking apparent motion and repetition blindness. Psychological Science, 8, 7479.

Crowder, R. G. (1968). Intraserial repetition effects in immediate memory. Journal of Verbal Learning \& Verbal Behavior, 7, 446-451.

Ebbinghaus, H. (1913). Memory (H. A. Ruger \& C. E. Bussenius, Trans.). New York: Teachers College. (Original work published 1885)

FAgot, C., \& Pashler, H. (1995). Repetition blindness: Perception or memory failure? Journal of Experimental Psychology: Human Perception \& Performance, 21, 275-292.

Green, D. M., \& Swets, J. A. (1966). Signal detection theory and psychophysics. New York: Wiley.

HABER, R. N. (1965). The effect of prior knowledge of the stimulus on word recognition processes. Journal of Experimental Psychology, 69, 282-286.

Haber, R. N., \& Hershenson, M. (1965). The effects of repeated brief exposures on the growth of a percept. Journal of Experimental Psychology, 69, 40-46.

HochHaus, L., \& Johnston, J. C. (1996). Perceptual repetition blindness effects. Journal of Experimental Psychology: Human Perception \& Performance, 22, 355-366.

HochHaus, L., \& Marohn, K. M. (1991). Repetition blindness depends on perceptual capture and token individuation failure. Journal of Experimental Psychology: Human Perception \& Performance, 17, 422432.

KANWISHER, N. G. (1987). Repetition blindness: Type recognition without token individuation. Cognition, 27, 117-143.

KANwisher, N. G. (1991). Repetition blindness and illusory conjunctions: Errors in binding visual types with visual tokens. Journal of Ex- perimental Psychology: Human Perception \& Performance, 17, 404421.

KANWISHER, N. G., DrIVER,J., \& MACHAdO,L. (1994). Spatial repetition blindness for color and shape. Canadian Psychology, 35, 105-106.

Kanwisher, N. G., Driver, J., \& Machado, L. (1995). Spatial repetition blindness is modulated by selective attention to color and shape. Cognitive Psychology, 29, 303-337.

Kanwisher, N. G., Kim, J. W., \& Wickens, T. D. (1996). Signal detection analyses of repetition blindness. Journal of Experimental Psychology: Human Perception \& Performance, 22, 1249-1260.

Keele, S. W. (1969). Repetition effect: A memory-dependent process. Journal of Experimental Psychology, 80, 243-248.

Lewandowsky, S., Neely, J. H., Ver Wy s, C. A., \& Amos, A. (1997, November). Repetition blindness under serial versus paired encoding. Poster presented at the 38th Annual Meeting of the Psychonomic Society, Philadelphia.

LowE, D. G. (1979). Strategies, context, and the mechanism of response inhibition. Memory \& Cognition, 7, 382-389.

Luo, C. R, \& CARAmazza, A. (1995). Repetition blindness under minimum memory load: Effects of spatial and temporal proximity and the encoding effectiveness of the first item. Perception \& Psychophysics, 57, 1053-1064.

Macmillan, N. A., \& Creelman, C. D. (1991). Detection theory: A user's guide. Cambridge: Cambridge University Press.

Morris, C. D., Bransford, J. D., \& FranKs, J. J. (1977). Levels of processing versus transfer appropriate processing. Journal of Verbal Learning \& Verbal Behavior, 16, 519-533.

Murdock, B. B. (1974). Human memory: Theory and data. Hillsdale, NJ: Erlbaum.

NeILL, W. T. (1977). Inhibitory and facilitatory processes in selective attention. Journal of Experimental Psychology: Human Perception \& Performance, 3, 444-450.

NeILL, W. T. (1985). Levels of processing in disruptive effects of prior information. Memory \& Cognition, 13, 477-484.

NeILL, W. T., \& MATHIS, K. M. (1998). Transfer-inappropriate processing: Negative priming and related phenomena. In D. L. Medin (Ed.), The psychology oflearning and motivation: Advances in research and theory (Vol. 38, pp. 1-44). San Diego: Academic Press.

Neill, W. T., Neely, J. H., Hutchison, K. A., Kahan, T. A., \& VerWys, C. A (2002). Repetition blindness, forward and backward. Journal of Experimental Psychology: Human Perception \& Performance, 28, 137-149.

Neill, W. T., \& Walling, J. R. (1981). Disruptive effects of prior information on tachistoscopic recognition. Memory \& Cognition, 9 , 217-224.

NeILl, W. T., \& Westberry, R. L. (1987). Selective attention and the suppression of cognitive noise. Journal of Experimental Psychology: Learning, Memory, \& Cognition, 13, 327-334.

PARK, J., \& KANWISHER, N. G. (1994). Determinants of repetition blindness. Journal of Experimental Psychology: Human Perception \& Performance, 20, 500-519.

RANSCHBURG, P. (1902). Über Hemmung gleichzeitiger Reizwirkungen [On inhibition of simultaneous stimulus effects]. Zeitschrift für Psychologie, 30, 39-86.

Roediger, H. L., III, \& Blaxton, T. A. (1987). Retrieval modes produce dissociations in memory for surface information. In D. S. Gorfein \& R. R. Hoffman (Eds.), Memory and learning: The Ebbinghaus centennial conference (pp. 349-379). Hillsdale, NJ: Erlbaum.

SCHNEIDER, W. (1988). Micro Experimental Laboratory: An integrated system for IBM PC compatibles. Behavior Research Methods, Instruments, \& Computers, 20, 206-217.

Smith, E. E., Haviland, S. E., Reder, L. M., Brownell, H., \& ADAMs, N. (1976). When preparation fails: Disruptive effects of prior information on perceptual recognition. Journal of Experimental Psychology: Human Perception \& Performance, 2, 151-161.

TIPPER, S. P. (1985). The negative priming effect: Inhibitory priming by ignored objects. Quarterly Journal of Experimental Psychology, 37A, 571-590.

TiPPER, S. P., \& CRANSTON, M. (1985). Selective attention and priming: Inhibitory and facilitatory effects of ignored primes. Quarterly Journal of Experimental Psychology, 37A, 591-611. 
Whittlesea, B. W. A., Dorken, M. D., \& Podrouzek, K. W. (1995). Repeated events in rapid lists: Pt. I. Encoding and representation. Journal of Experimental Psychology: Learning, Memory, \& Cognition, 21, 1670-1688.

Whittlesea, B. W. A., \& Podrouzek, K. W. (1995). Repeated events in rapid lists: Pt. II. Remembering repetitions. Journal of Experimental Psychology: Learning, Memory, \& Cognition, 21, 1689-1697.

\section{NOTES}

1. Several similar studies warrant mention. Fagot and Pashler (1995, Experiment 3) conducted an experiment in which subjects were informed of two critical target letters (e.g., A and B) at the beginning of a rapid letter sequence. Unlike Kanwisher et al. (1996), there were always two target letters in the letter stream, and subjects were required to report the specific sequence of targets-for example, A-A, A-B, B-A or B-B. An SDT analysis suggested an actually greater $d^{\prime}$ for repetitions but a bias toward reporting nonrepetitions. However, because the subjects had to report the specific identities, this experiment does not follow the logic of the counting task. Kanwisher et al. (1996) pointed out that repetitions were given an unfair advantage in this task: If the subjects detected two different targets, they had to distinguish the order in which they occurred, whereas this would be unnecessary if the subjects detected repeated targets. Kanwisher et al. (1996) also argued that Fagot and Pashler made erroneous assumptions in their SDT analysis.

Whittlesea and Podrouzek (1995, Experiment 2) presented sevenword lists that sometimes contained a repetition. Subjects were required to report whether a repetition had occurred, as well as to recall the whole list on each trial. One group reported whether a repetition occurred before recalling the list, whereas another group recalled the list first. A measure related to $d^{\prime}\left(d_{L}\right)$ indicated that repetition detection was better following recall than preceding it. Although this result indicates that memory retrieval can affect the report of repetitions, there was no comparable unrepeated condition requiring a count of "two." Thus, it cannot be determined whether RB occurred for either condition in this task.

Arnell and Jolicœur (1997) required subjects to count animal pictures embedded in a series of line drawings. In fact, there were always two animal pictures, either identical or nonidentical. In their Experiment 2, subjects were required to report the total frequency of animal pictures $(0,1$,
$2,3$, or 4$)$, rather than the frequency of individual pictures. The percentage of "two" responses was lower for repeated pictures (62\%) than for nonrepeated pictures (69\%). However, this design could not distinguish between discriminability and bias effects, because the actual number of pictures was not varied. Indeed, the fact that the subjects made a fairly high number of "three" and "four" responses (12\% in repeated trials, $17 \%$ in nonrepeated trials) suggests that subjects' guessing criteria could contribute to the pattern of results.

2. Exposure duration was manipulated here only to facilitate comparison with Experiment 2, which predated Experiment 1.

3 . The SDT measures $d^{\prime}$ and $\beta$ assume that the noise and signal + noise distributions are both normal and equal-variance. Other measures of discriminability and bias are possible, given different distributional assumptions. We focus on $d^{\prime}$ here because that is the measure emphasized by Park and Kanwisher (1994) and Kanwisher et al. (1996). They also required subjects to give confidence ratings on each response, to calculate $\Delta M$, which does not assume equal-variance distributions. This measure yielded results similar to $d^{\prime}$. (Although Kanwisher et al., 1996, did not report the condition means for $\Delta M$, their Appendix B indicates that the "substantive differences for the two measures were approximately the same," p. 1260). Kanwisher et al. (1996) also reported high-threshold alpha, which is essentially a linear correction for guessing. This measure, too, yielded results similar to $d^{\prime}$.

4. All analyses of response bias were actually conducted on $\log (\beta)$ for individual subjects, rather than $\beta$. The likelihood-ratio $\beta$ ranges from 0 to 1 for positive response bias, but from 1 to infinity for negative response bias, which introduces severe heterogeneity of variance across the scale. $\log (\beta)$, however, is symmetrical about 0 for positive and negative biases. We report the means transformed to $\beta$, because that measure is more likely to be familiar to readers; however, $M S_{\mathrm{e}}$ is reported for the $\log (\beta)$ analysis.

5. It should be noted that the discrimination of two identical targets from two nonidentical targets is irrelevant to testing $\mathrm{RB}$, because either veridical perception or detection of only one of two identical targets enables this discrimination.

Manuscript received September 20, 2000; revision accepted for publication October 10, 2001.) 